\title{
Identification and authentication of Rosa species through development of species-specific SCAR marker(s)
}

\author{
K.M.I. Bashir ${ }^{1}$, F.S. Awan ${ }^{1}$, I.A. Khan ${ }^{1}$, A.I. Khan ${ }^{1}$ and M. Usman ${ }^{2}$ \\ ${ }^{1}$ Centre of Agricultural Biochemistry and Biotechnology, \\ University of Agriculture, Faisalabad, Pakistan \\ ${ }^{2}$ Institute of Horticultural Sciences, University of Agriculture, \\ Faisalabad, Pakistan \\ Corresponding author: K.M.I. Bashir \\ E-mail: imranagrarian3@gmail.com
}

Genet. Mol. Res. 13 (2): 4130-4139 (2014)

Received July 1, 2013

Accepted February 27, 2014

Published May 30, 2014

DOI http://dx.doi.org/10.4238/2014.May.30.8

\begin{abstract}
Roses (Rosa indica) belong to one of the most crucial groups of plants in the floriculture industry. Rosa species have special fragrances of interest to the perfume and pharmaceutical industries. The genetic diversity of plants based on morphological characteristics is difficult to measure under natural conditions due to the influence of environmental factors, which is why a reliable fingerprinting method was developed to overcome this problem. The development of molecular markers will enable the identification of Rosa species. In the present study, randomly amplified polymorphic DNA (RAPD) analysis was done on four Rosa species, Rosa gruss-an-teplitz (Surkha), Rosa bourboniana, Rosa centifolia, and Rosa damascena. A polymorphic RAPD fragment of $391 \mathrm{bp}$ was detected in $R$. bourboniana, which was cloned, purified, sequenced, and used to design a pair of speciesspecific sequence-characterized amplified region (SCAR) primers (forward and reverse). These SCAR primers were used to amplify the specific regions of the rose genome. These PCR amplifications with
\end{abstract}


specific primers are less sensitive to reaction conditions, and due to their high reproducibility, these species-specific SCAR primers can be used for marker-assisted selection and identification of Rosa species.

Key words: Rose; Cloning; Sequencing; RAPD; SCAR

\section{INTRODUCTION}

Roses belong to the most important groups of ornamental plants. Roses have been admired for their beauty and fragrance and have had a unique place in people's hearts since their first cultivation, approximately 5000 years ago. Roses were cultivated for the first time in China, Northern Africa and Western Asia, but now, they are cultivated throughout the world (Gudin, 2000).

The five major classes of roses, i.e., Gallica (Rosa gallica), Alba (R. alba), Damask (R. damascena), Centifolia (R. centifolia), and Moss ( $R$. centifolia moscosa), had emerged by the end of eighteenth century. These five classes had many characteristics in common, such as fragrance, flower number, flower color, winter hardiness, and resistance to certain diseases (namely black spot and rust). On the basis of these similarities, they were grouped as old European roses (Debener and Linde, 2009). Only few of the cultivated rose varieties reveal the marked aroma that attracts the world perfumeries. Previous studies have shown that the identification of rose cultivars and species was based mostly on morphological characters such as growth habit or floral morphology (Mohapatra and Rout, 2006).

Approximately 60 years have passed since the structure and biological function of the DNA was discovered and nucleic acid-based inspections started spreading. Currently, researchers are regularly using these methods in various fields of biological sciences. Genetic studies of roses have been conducted with the help of molecular markers (Debener and Mattiesch, 1999; Rajapakse et al., 2001; Crespel et al., 2002).

In roses, DNA markers have been used for cultivar identification and to study genetic diversity (Debener, 2002; Esselink et al., 2003). Randomly amplified polymorphic DNA (RAPD) and restriction fragment length polymorphism (RFLP) markers have been used to determine genetic relatedness of siblings (Rameau et al., 1998), for cultivar identification (Hubbard et al., 1992), phylogenetic analyses within and between Rosa species and mapping rose genes against powdery mildew/black spot resistance (Linde et al., 2004). However, disease resistance maps have a medium marker density and can be used as tools for genetic diversity studies and molecular breeding of rose plants (Rajapakse, 2003). RFLP and simple sequence repeats have been preferred due to their instructiveness. RAPD markers are still used due to simplicity, low cost and lower infrastructure requirements (William et al., 1990; Upadhya et al., 2004). However, RAPDs lack reproducibility due to mismatched annealing and so are not usually preferred.

The sequence-characterized amplified region (SCAR) technique was developed by Paran and Michelmore (1993). RAPD and RFLP-derived SCAR markers have been significantly used for plant identification at inter- and intraspecies levels (Roh et al., 2006; Choi et al., 2008). Random primers are used for the identification of the SCAR marker, which is actually the DNA fragment of the same genome. The conversion of these markers into SCAR markers greatly improves the reliability and reproducibility of PCR amplifications, especially RAPD analysis. 
SCAR primers have been reported in many crops, including apple (De Clercq et al., 2003), papaya (Deputy et al., 2002), cowpea (Boukar et al., 2004), brassica (Piquemal et al., 2005), strawberry (Rugienius et al., 2006), bamboo (Das et al., 2005), buffelgrass (Dwivedi et al., 2007), and citrus (Ping et al., 2009). While the literature on the development of SCAR markers in roses is extremely meager, there are a few reports to date. Linde et al. (2004) and Yan et al. (2005) developed SCAR markers in roses by utilizing RFLP markers. Whitaker et al. (2010) reported SCAR marker development for a specific race of tetraploid roses against black spot resistance, and Riaz et al. (2012) reported SCAR marker development for R. centifolia. But, there are still no reports available on SCAR marker development from RAPD in R. bourboniana .

The intention of the present study was to convert the dominant RAPD marker(s) into the co-dominant SCAR marker(s). SCARs detect a single locus as compared to RAPD markers. In the present study, a pair of species-specific SCAR primers was found to be co-dominant and could subsequently be utilized to identify particular rose species. Their amplification is less sensitive to reaction conditions, and due to their high reproducibility, SCARs can be used for extensive marker-assisted selections.

\section{MATERIAL AND METHODS}

\section{Plant material}

Leaves of four Rosa species, R. gruss-an-teplitz (Surkha), R. bourboniana, R. centifolia, and $R$. damascena, were collected for genomic DNA isolation. DNA was isolated by using the Doyle and Dolly (1990) DNA isolation method with slight modification. The DNA quantities were then determined using a NanoPhotometer, and $15 \mathrm{ng}$ were used as working dilutions for RAPD-PCR.

\section{RAPD (PCR) amplification}

RAPD-PCR based primers were used to amplify the DNA of four Rosa species. A total of 19 RAPD (Table 1) primers (GL-RAPD decamer) custom synthesized by GENELINK (Catalog No. 40-0001-01; Hawthorne, NY, USA) were used, and one primer, GLB-15, identified a polymorphic band in $R$. bourboniana. The PCR product was electrophoresed on a $1.2 \%$ agarose gel in TBE buffer. The polymorphic fragment, GLB- $15_{391}$, was eluted from the gel with the help of the QIAGEN gel extraction kit (50) (Catalog No. 28704; QIAGEN, Hilden, Germany).

\section{Cloning of GLB-15}

The eluted fragment was directly cloned into the PTZ57R/T cloning vector provided by the TA cloning kit from Fermentas (UAB), Vilnius, Lithuania. Escherichia coli DH5 $\alpha$ competent cells were transformed by heat shock method by following the transformation protocol as modified by Froger and Hall (2007). Plasmid DNA from the transformed E. coli cells was isolated by using the Sambrook and Russel (2001) plasmid isolation method with slight modifications. After quantification of the plasmids using a NanoPhotometer, two separate restriction digestions were performed on the basis of the plasmid MCS sequence. The first digestion with Pst 1 and the other as double digestion with Pst 1 and EcoR 1 in combination, and the restriction results were checked on a $1.2 \%$ agarose gel. 


\begin{tabular}{lll}
\multicolumn{2}{c}{ Table 1. List of the RAPD primers. } & \\
\hline Sr. No. & Primer name & Primer sequence \\
\hline 1 & GL Decamer B-07 & GGTGACGCAG \\
2 & GL Decamer B-08 & GTCCACACGG \\
3 & GL Decamer B-10 & CTGCTGGGAC \\
4 & GL Decamer B-12 & CCTTGACGCA \\
5 & GL Decamer B-15 & GGAGGGTGTT \\
6 & GL Decamer B-16 & TTTGCCGGA \\
7 & GL Decamer I-02 & GGAGGAGAGG \\
8 & GL Decamer K-04 & CCGCCCAAAC \\
9 & GL Decamer K-05 & TCTGTCGAGG \\
10 & GL Decamer K-08 & GAACACTGGG \\
11 & GL Decamer K-12 & TGGCCCTCAC \\
12 & GL Decamer K-16 & GAGCGTCGAA \\
13 & GL Decamer K-19 & CACAGGCGGA \\
14 & GL Decamer K-20 & GTGTCGCGAG \\
15 & GL Decamer L-02 & TGGGCGTCAA \\
16 & GL Decamer L-04 & GACTGCACAC \\
17 & GL Decamer L-07 & AGGCGGGAAC \\
18 & GL Decamer L-08 & AGCAGGTGGA \\
19 & GL Decamer L-11 & ACGATGAGCC \\
\hline
\end{tabular}

\section{DNA sequencing}

The complete nucleotide sequence of the cloned fragment GLB- $15_{391}$ was determined by the dideoxynucleotide chain termination sequencing method using the PCR-based BIG DYE kit with M-13 primers. Reaction products were resolved on an ABI PRISM ${ }^{\circledR} 3100$ automated genetic analyzer (Applied Biosystems, USA). Sequence information was saved, compiled and analyzed by the ABI PRISM ${ }^{\circledR}$ GeneScan Analysis Software version 3.7 (Sgueglia et al., 2003).

\section{Primer designing}

On the basis of the sequence of the cloned fragment, a pair of SCAR primers (forward and reverse) ranging from 20 to $22 \mathrm{bp}$ in length was designed with the help of the Primer-3 software by keeping the annealing temperatures of the primers between $55^{\circ}$ and $60^{\circ} \mathrm{C}$. GC content and the annealing temperature were calculated by using the JUTBIO hosted tools.

\section{RESULTS}

\section{RAPD-PCR}

RAPD markers were utilized to identify the polymorphic fragment(s) in Rosa species. Only one primer GLB-15 produced a polymorphic fragment of approximately $391 \mathrm{bp}$ in $R$. bourboniana and was found absent in the other three Rosa species under study (Figure 1).

\section{Elution of the polymorphic fragment}

The specific 391-bp fragment was eluted from the gel with a QIAGEN gel extraction kit (50) and was confirmed on a $1.2 \%$ agarose gel. The elution of the same fragment was per- 
formed twice to eliminate any handling error, and the same light single band of $391 \mathrm{bp}$ was found in both cases, which verified the successful elution of the RAPD-PCR fragment (Figure 2).

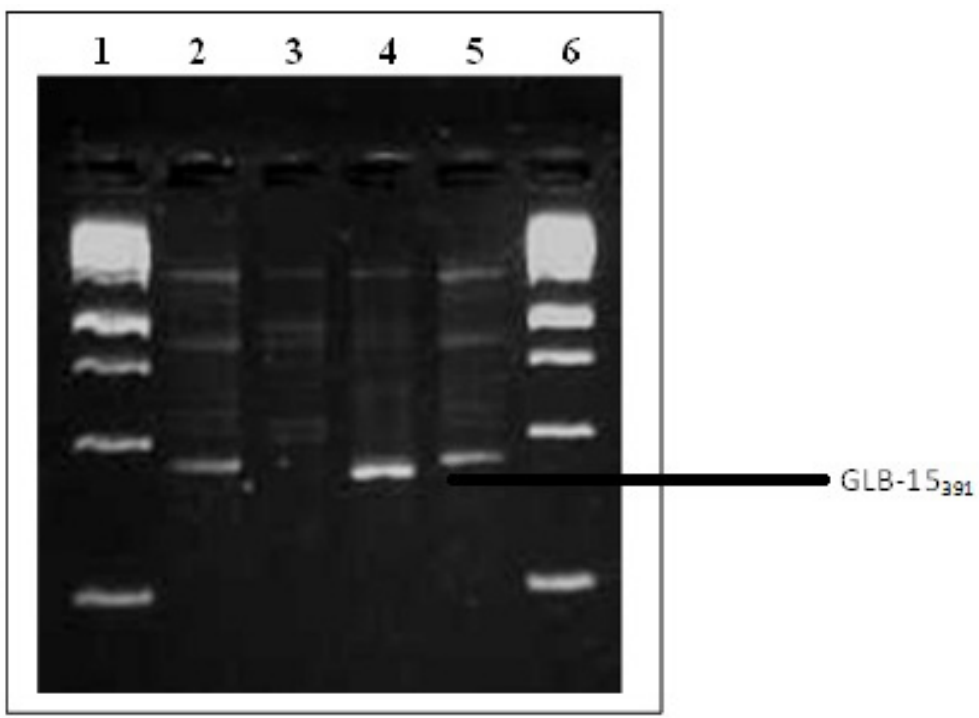

Figure 1. RAPD-PCR. Lanes 1 and $6=1$-kb DNA ladder; lane $2=$ Rosa centifolia; lane $3=$ Rosa grus-en-teplitz; lane $4=$ Rosa bourboniana; lane $5=$ Rosa damascena .

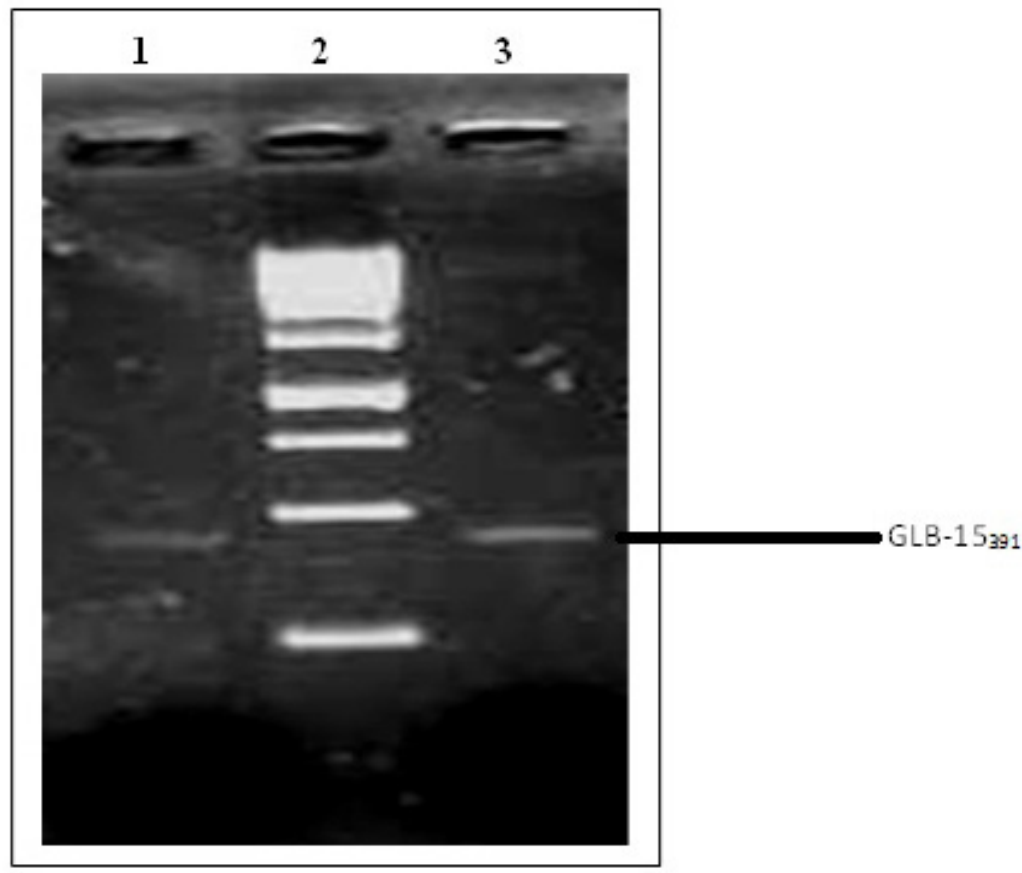

Figure 2. Eluted fragment. Lanes 1 and $3=$ eluted DNA fragment; lane $2=1-\mathrm{kb}$ DNA ladder. 


\section{Re-amplification of the PCR product}

Re-amplification of the RAPD (PCR) fragment was done by using the eluted fragment as the template and keeping the same reaction conditions. The bright single fragment of 391 bp was amplified in three reactions with GLB-15 RAPD primer (Figure 3). It confirmed no internal primer binding sites for GLB-15 primer in the 391-bp fragment.

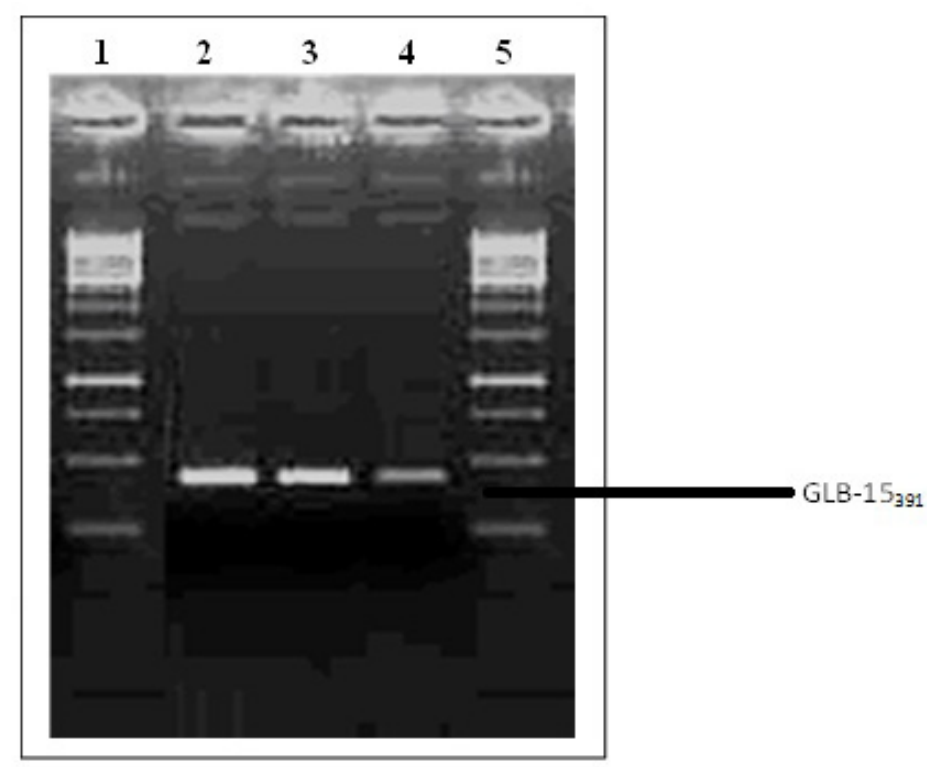

Figure 3. Re-amplified PCR product. Lanes 1 and 5=1-kb DNA ladder. Lanes 2-4 = re-amplified PCR product.

\section{Cloning of the PCR product}

The eluted fragment was directly cloned into the TA cloning vector PTZ57R/T. After successful transformation, the plasmids were isolated and the quality and quantity of isolated plasmids were checked on a 1.2\% agarose gel (Figure 4). The digestion of the plasmids with Pst 1 , confirmed the successful cloning of GLB-15 ${ }_{391}$ in PTZ57R/T (Figure 5). Six digested plasmids were run on a gel and four of them showed the proper digestion with the specific enzyme. The enzyme EcoR1 used in combination with Pst 1 gave complete digestion of the plasmid and the 391-bp fragment was confirmed on a 1.2\% agarose gel (Figure 6).

\section{Sequencing}

The cloned fragment was sequenced with an ABI PRISM ${ }^{\circledR} 3100$ automated genetic analyzer (Applied Biosystems, USA) by using fluorescence-based capillary electrophoresis platform with the vector specific M-13 primers, and the 391-bp fragment was confirmed. Since the fragment size was approximately $391 \mathrm{bp}$, the sequencing results were easily obtained.

The sequencing results were as follows: 
ACTAGGAATTAGGGTGTCTAGGTGTTGCAGGGTGTTGCAGGGTGTTGGAGGGCTA AGAGTGGTACATCTGCTTGTATGGTGTGGCAACCATTTCTTTTCCAATCATTGCCT GTTCATATTCTCTATAATTCCTTCAAAAAATTGTTCAATGTTGAAACCGTAGAGGAC TCTGAAATTTCATTTCTTTCGCTGTCCTCTACTTTAGTTTACTCTTGTATTGGATGTC TTGGCTCTTTCCAGCTCTAATGGTCAACCATGGATACTTACAATACTACACATCTTT CATAACAGCAAATGTACTATCTCTTTTAAAAATATAAAAGCTACATTGACCAATTTTC CAAATCGTACAACCTAAACACCCCAACACTCCTGAGACTCAAGACCTCAG.

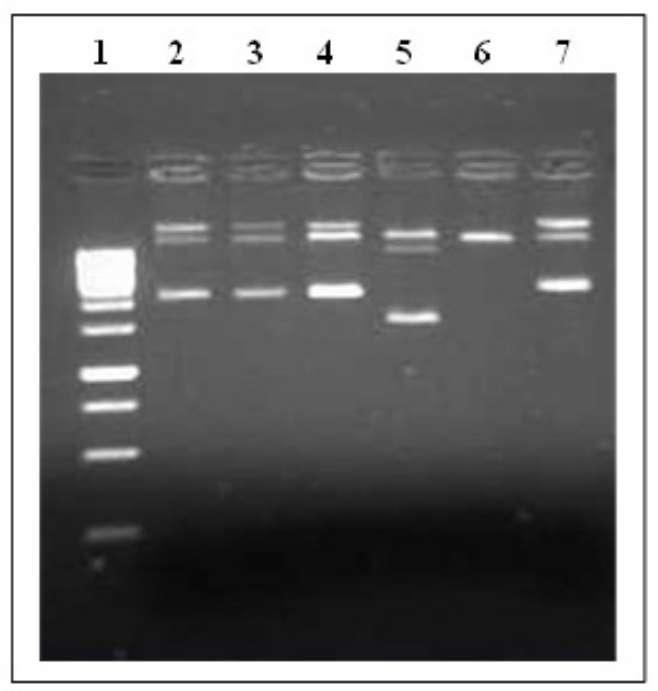

Figure 4. Miniprep plasmids. Lane 1=1-kb DNA ladder; lanes 2-7= miniprep samples (undigested).

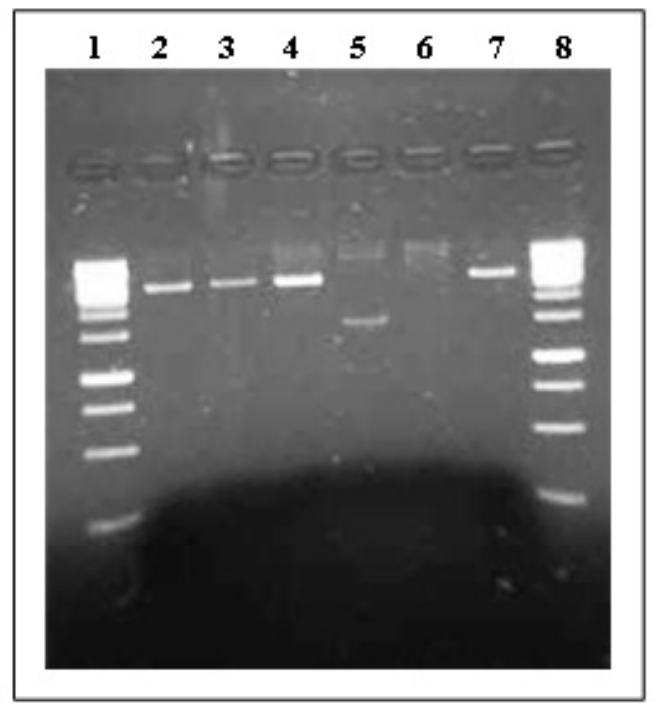

Figure 5. Single digested plasmids. Lanes 1 and $8=1-\mathrm{kb}$ DNA ladder; lanes 2-7 = single digestion (Pst 1$)$. 


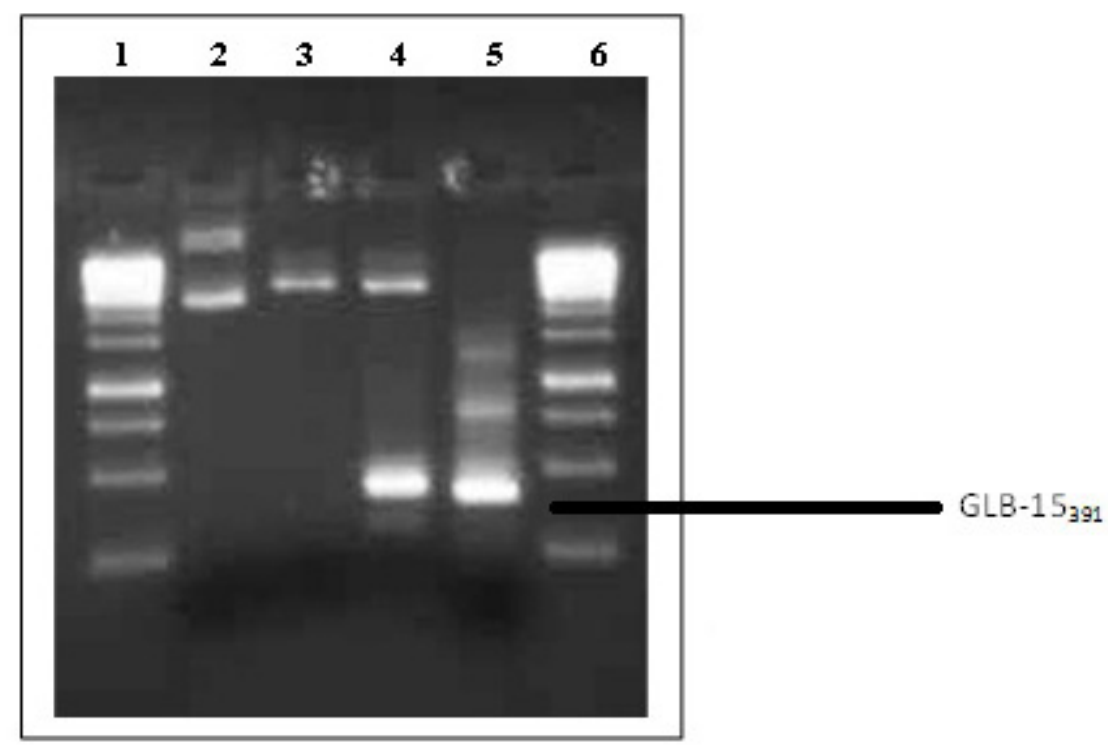

Figure 6. Double digested plasmids. Lanes 1 and $6=1$-kb DNA ladder; lane $2=$ undigested plasmids; lane $3=$ single digestion with Pst 1 ; lane $4=$ double digestion (Pst 1 and EcoR1); lane $5=$ PCR product.

\section{Primer designing}

After sequencing of the DNA fragment, a pair of species-specific SCAR primers (forward: CTAGGAATTAGGGTGTCTAGG and reverse: CTGAGGTCTTGAGTCTCAGG) was designed, on the basis of the flanking regions of the cloned fragment. The 20- to 22-bp primers were designed by the Primer-3 software and keeping their annealing temperatures between $55^{\circ}$ and $60^{\circ} \mathrm{C}$, so that at this temperature they would anneal in the genomic regions and would produce single locus amplifications (Rugienius et al., 2006). GC content and the annealing temperature were calculated by using the JUTBIO hosted tools.

\section{DISCUSSION}

In the present research, four indigenous Rosa species of Pakistan were investigated for the detection of polymorphism by RAPD analysis. The Rosa germplasm generally lacks genetic variability except for a few morphological characters. Furthermore, the complex or secret breeding history of most rose cultivars make it more difficult or impossible to estimate the genetic relationships of rose varieties and the genetic diversity within rose groups on the basis of pedigree data (Sgueglia et al., 2003).

RAPD technology has been broadly used to identify the genetic diversity between complex genome species (Dhanya and Sasikumar, 2010). RAPD became more advantageous due to its simplicity and rapidity, requirement of low amounts of genomic DNA, and no need of prior genetic information (Williams et al., 1990). In recent years, many technical drawbacks of RAPD methods have limited its widespread use as a genetic marker. The reproducibility of RAPD is affected by DNA quality, primer and template concentrations, different thermal 
cyclers and even different sources of DNA polymerase (Ellsworth et al., 1993). Subsequently, the conversion of RAPD to SCAR markers (Paran and Michelmore, 1993) by developing longer and hence more specific primers from RAPD sequences has significantly improved the reproducibility and reliability of PCR assays (Rugienius et al., 2006). SCAR markers have many advantages as compared to RAPD and RFLP makers. SCARs have characteristics favorable for marker-assisted selection, and like some other PCR-based markers, they can be rapidly and easily used in species identification and accurately for genotype authentication.

In the present research, a polymorphic RAPD fragment of $391 \mathrm{bp}$ was identified in Rosa bourboniana and successfully cloned into a PTZ57R/T cloning vector. Due to the small size of the fragment, the re-amplification of the RAPD fragment was done, and no internal binding sites for the RAPD primers were found. After end-sequencing of the fragment cloned, a pair of SCAR primers (forward and reverse) was designed. These primers can be easily used for early identification and screening of $R$. bourboniana. The results also provide the basis for the identification and authentication of other Rosa species and for developing their fingerprints.

\section{REFERENCES}

Boukar O, Kong L, Singh BB, Murdock L, et al. (2004). AFLP and AFLP-derived SCAR markers associated with Striga gesnerioides resistance in cowpea. Crop Sci. 44: 1259-1264.

Choi YE, Ahn CH, Kim BB and Yoon ES (2008). Development of species specific AFLP-derived SCAR marker for authentication of Panax japonicus C.A. MEYER. Biol. Pharm. Bull. 31: 135-138.

Crespel L, Chirollet M, Durel E, Zhang D, et al. (2002). Mapping of qualitative and quantitative phenotypic traits in Rosa using AFLP markers. Theor. Appl. Genet. 105: 1207-1214.

Das M, Bhattacharya S and Pal A (2005). Generation and characterization of SCARs by cloning and sequencing of RAPD products: a strategy for species-specific marker development in bamboo. Ann. Bot. 95: 835-841.

De Clercq D, Cognet S, Pujol M, Lepoivre P, et al. (2003). Development of a SCAR marker and a semi-selective medium for specific quantification of Pichia anomala strain K on apple fruit surfaces. Postharvest Biol. Technol. 29: 237-247.

Debener T (2002). Molecular Markers as a Tool for Analyses of Genetic Relatedness and Selection in Ornamentals. In: Breeding for Ornamentals: Classical and Molecular Approaches (Vainstein A, ed.). Dordrecht, 329-345.

Debener T and Mattiesch L (1999). Construction of genetic linkage map for roses using RAPD and AFLP markers. Theor. Appl. Genet. 99: 891-899.

Debener T and Linde M (2009). Exploring complex ornamental genomes: The rose as a model plant. Crit. Rev. Plant Sci. 28: $267-280$.

Deputy JC, Ming R, Ma H, Liu Z, et al. (2002). Molecular markers for sex determination in papaya (Carica papaya L.). Theor. Appl. Genet. 106: 107-111.

Dhanya K and Sasikumar B (2010). Molecular marker based adulteration detection in traded food and agricultural commodities of plant origin with special reference to spices. Curr. Trends Biotechnol. Pharm. 4: 454-489.

Doyle JJ and Dolly JL (1990). Isolation of plant DNA from fresh tissue. Focus 12: 13-15.

Dwivedi KK, Bhat SR, Bhat V and Bhat BV (2007). Identification of a SCAR marker linked to apomixes in buffel grass (Cenchrus ciliaris L.). Plant Sci. 172: 788-795.

Ellsworth DL, Rittenhouse KD and Honeycutt RL (1993). Artifactual variation in randomly amplified polymorphic DNA banding patterns. Biotechniques 14: 214-217.

Esselink GD, Smulders MJ and Vosman B (2003). Identification of cut rose (Rosa hybrida) and rootstock varieties using robust sequence tagged microsatellite site markers. Theor. Appl. Genet. 106: 277-286.

Froger A and Hall JE (2007). Transformation of plasmid DNA into E. coli using the heat shock method. J. Vis. Exp. 253.

Gudin S (2000). Rose: genetics and breeding. Avenue escadrille Normandie-Neimen, 13397 Marseille, Cedex 20, France. Plant Breed. Rev. 8: 159-189.

Hubbard M, Kelly J, Rajapakse S and Abbott A (1992). Restriction fragment length polymorphism in rose and their use for cultivar identification. HortScience 27: 172-173.

Linde M, Mattiesch L and Debener T (2004). Rpp1, a dominant gene providing race-specific resistance to rose powdery mildew (Podosphaera pannosa): molecular mapping, SCAR development and confirmation of disease resistance 
data. Theor. Appl. Genet. 109: 1261-1266.

Mohapatra A and Rout GR (2006). Optimization of primer screening for evaluation of genetic relationship in rose cultivars. Biol. Plantarum 50: 295-299.

Paran I and Michelmore RW (1993). Development of reliable PCR-based markers linked to downy mildew resistance genes in lettuce. Theor. Appl. Genet. 85: 985-993.

Ping XJ, Ligeng C, Ming X and Hailin L (2009). Identification of AFLP fragments linked to seedlessness in Ponkan mandarin (Citrus reticulate Blanco) and conversion to SCAR markers. Sci. Hort. 121: 505-510.

Piquemal J, Cinquin E, Couton F, Rondeau C, et al. (2005). Construction of an oilseed rape (Brassica napus L.) genetic map with SSR markers. Theor. Appl. Genet. 111: 1514-1523.

Rajapakse S (2003). Gene Mapping. In: Encyclopedia of Rose Sciences (Roberts A, Debener T and Gudin S, eds.). Elsevier, Oxford, 326-334.

Rajapakse S, Byrne DH, Zhang L and Anderson N (2001). Two genetic linkage maps of tetraploid roses. Theor. Appl. Genet. 103: 575-583.

Rameau C, Denouse D, Fraval F and Haurogne K (1998). Genetic mapping in pea. Identification of RAPD and SCAR markers linked to genes affecting plant architecture. Theor. Appl. Genet. 97: 916-928.

Riaz S, Sadia B, Awan FS, Khan IA, et al. (2012). Development of a species-specific sequence-characterized amplified region marker for roses. Genet. Mol. Res. 11: 440-447.

Roh MS, Lee AK, Suh JK and Slovin J (2006). Confirmation of cross-pollination of Ardisia crenata by sequencecharacterized amplified region (SCAR) markers. Sci. Hort. 109: 361-367.

Rugienius R, Siksnianas T, Stanys V, Gelvonauskiene D and Bendokas V (2006). Use of RAPD and SCAR markers for identification of Strawberry genotypes carrying red stele (Phytophtora fragariae) resistance gene Rpf1. Agron. Res. 4: 335-339.

Sambrook J and Russell DW (2001). Molecular Cloning: A Laboratory Manual, Third edn. Cold Spring Harbor Laboratory, New York.

Sgueglia JB, Geiger S and Davis J (2003). Precision studies using the ABI prism 3100 genetic analyzer for forensic DNA analysis. Anal. Bioanal. Chem. 376: 1247-1254.

Upadhya A, Jayadev K, Manimekalai R and Parthasarathy VA (2004). Genetic relationship and diversity in Indian account accessions based on RAPD markers. Sci. Hort. 99: 353-362.

Whitaker VM, Bradeen JM, Debener T, Biber A, et al. (2010). Rdr3, a novel locus conferring black spot disease resistance in tetraploid rose: genetic analysis, LRR profiling, and SCAR marker development. Theor. Appl. Genet. 120: 573-585.

Williams JG, Kubelik AR, Livak KJ, Rafalski JA, et al. (1990). DNA polymorphisms amplified by arbitrary primers are useful as genetic markers. Nucleic Acids Res. 18: 6531-6535.

Yan Z, Denneboom C, Hattendorf A, Dolstra O, et al. (2005). Construction of an integrated map of rose with AFLP, SSR, PK, RGA, RFLP, SCAR and morphological markers. Theor. Appl. Genet. 110: 766-777. 